\title{
The bone marrow microenvironment as a mediator of chemoresistance in acute lymphoblastic leukemia
}

\author{
Lauren K. Meyer, Michelle L. Hermiston \\ Department of Pediatrics, University of California, San Francisco, SF 94158, USA.
}

Correspondence to: Michelle L. Hermiston, Department of Pediatrics, University of California, San Francisco, SF 94158, USA. E-mail: michelle.hermiston@ucsf.edu

How to cite this article: Meyer LK, Hermiston ML. The bone marrow microenvironment as a mediator of chemoresistance in acute lymphoblastic leukemia. Cancer Drug Resist2019;2:1164-77. http://dx.doi.org/10.20517/cdr.2019.63

Received: 18 Jul 2019 First Decision: 2 Sep 2019 Revised: 12 Sep 2019 Accepted: 27 Sep 2019 Published: 19 Dec 2019

Science Editor: Frits Peters Copy Editor: Rong-Rong Dou Production Editor: Tian Zhang

\begin{abstract}
Acute lymphoblastic leukemia (ALL) is a malignancy of immature lymphoid cells that arises due to clonal expansion of cells that undergo developmental arrest and acquisition of pathogenic mutations. With the introduction of intensive multi-agent chemotherapeutic regimens, survival rates for ALL have improved dramatically over the past several decades, though survival rates for adult ALL continue to lag behind those of pediatric ALL. Resistance to chemotherapy remains a significant obstacle in the treatment of $A L L$, and chemoresistance due to molecular alterations within $A L L$ cells have been described. In addition to these cell-intrinsic factors, the bone marrow microenvironment has more recently been appreciated as a cell-extrinsic mediator of chemoresistance, and it is now known that stromal cells within the bone marrow microenvironment, through direct cell-cell interactions and through the release of lymphoid-acting soluble factors, contribute to ALL pathogenesis and chemoresistance. This review discusses mechanisms of chemoresistance mediated by factors within the bone marrow microenvironment and highlights novel therapeutic strategies that have been investigated to overcome chemoresistance in this context.
\end{abstract}

Keywords: Acute lymphoblastic leukemia, chemotherapy, chemoresistance, bone marrow microenvironment

\section{INTRODUCTION}

Acute lymphoblastic leukemia (ALL) is a malignancy of immature lymphoid cells of either the B-cell (B-ALL) or T-cell (T-ALL) lineage. ALL is the most common malignancy of childhood, and survival rates for children with ALL now approach $90 \%$ on modern treatment protocols, with even better survival rates for some molecular subtypes ${ }^{[1]}$. In contrast, survival rates for adult ALL remain lower at $30 \%-40 \%{ }^{[2]}$. In 
Table 1. Mechanisms of action of chemotherapeutic agents used in the treatment of acute lymphoblastic leukemia

\begin{tabular}{ll}
\hline Drug & \multicolumn{1}{c}{ Mechanism of action } \\
\hline Asparaginase & Cleaves exogenous asparagine to deprive ALL cells of this amino acid \\
Clofarabine & Purine nucleoside analog that inhibits synthesis and repair of DNA \\
Corticosteroids (prednisone or dexamethasone) & Glucocorticoid receptor agonists that induce pro-apoptotic effects in lymphoid cells \\
Daunorubicin and Doxorubicin & Anthracyclines that inhibit topoisomerases \\
Etoposide & Topoisomerase II inhibitor \\
Methotrexate & Antimetabolite that inhibits dihydrofolate reductase to inhibit synthesis of purine nucleotides \\
Vincristine & Microtubule-destabilizing agent \\
\hline
\end{tabular}

both contexts, improvements in survival were achieved in recent decades due in large part to the continued optimization of intensive multi-agent chemotherapy. The agents most commonly utilized in the treatment of ALL and their mechanisms of action are outlined in Table 1. Despite these improvements, patients with relapsed or refractory ALL continue to face inferior outcomes, underscoring the importance of studies aimed at understanding the drivers of chemoresistance and identifying novel therapeutic strategies to restore chemosensitivity.

The genomic landscapes of both pediatric and adult ALL have been extensively characterized ${ }^{[3-5]}$, and in many cases the presence of specific molecular or cytogenetic alterations can be correlated with prognosis or with resistance to a particular chemotherapeutic agent ${ }^{[6-10]}$. These genetic factors therefore represent cellintrinsic drivers of chemoresistance, and there have been considerable efforts to target these resistance mechanisms through the use of biologic and small molecule therapeutic agents. Equally important however are the complex and diverse cell-extrinsic factors that similarly modulate response to chemotherapy and clinical outcomes in ALL.

Specifically, both normal hematopoietic progenitor cells and ALL cells residing in the bone marrow receive key survival, proliferative, and homing signals from the bone marrow microenvironment. This microenvironment is classically subdivided into two distinct niches comprised of the endosteal niche and the vascular niche. In the endosteal niche, hematopoietic cells maintain direct cell-cell interactions with osteoblasts, which support hematopoietic cell survival and proliferation. The vascular niche consists of small sinusoidal blood vessels within the marrow cavity, which ensure the movement of oxygen, nutrients, and cellular homing factors in and out of the bone marrow, thereby supporting hematopoietic cell function and maintaining communication with other tissue types ${ }^{[11]}$. Given these abundant interactions between the bone marrow microenvironment and ALL cells, there is significant interest in elucidating the microenvironmental drivers of chemoresistance in ALL and in identifying novel therapeutic strategies to overcome these cellextrinsic mechanisms of chemoresistance ${ }^{[12-14]}$.

\section{CELL-CELL INTERACTIONS IN THE BONE MARROW MICROENVIRONMENT}

A number of model systems of have been investigated for their ability to facilitate the ex vivo analysis of primary patient ALL cells, which demonstrate a high rate of spontaneous apoptosis under standard cell culture conditions. In particular, it has been shown that co-culture with bone marrow stromal cells (BMSCs) significantly enhances the survival of leukemic blasts ex $v i v{ }^{[15]}$. Specifically, this survival effect is often dependent upon direct contact with BMSCs, as BMSC conditioned media is insufficient to mediate the same improvement in cell survival ${ }^{[16]}$. These studies highlight the importance of cell-cell interactions with BMSCs for the survival and proliferation of ALL cells, and a number of studies, many of which are described below, have demonstrated that these cell-cell interactions mediate chemoresistance.

\section{Integrins}

Integrins are a class of transmembrane cell adhesion receptors that make up a critical component of the extracellular matrix (ECM). Integrins are comprised of heterodimers of a number of distinct $\alpha$ and $\beta$ 
subunits and each have specific ligand-binding capabilities, determined predominantly by the identity of the $\alpha$ subunit. Upon engagement by extracellular stimuli, activated integrins mediate a variety of signal transduction events leading to modulation of a wide range of cellular processes, including cell survival, gene expression, and cell motility ${ }^{[17]}$.

Despite the ubiquitous expression of this class of cell adhesion receptors throughout all tissue types, distinct integrin heterodimers demonstrate tissue-specific expression patterns. The $\beta 1$-containing integrins, collectively known as very-late activation antigens (VLAs), heterodimerize with $\alpha 4$ subunits (CD49b) to form VLA-4. VLA-4 is highly expressed on hematopoietic progenitors and more mature blood cells, including healthy leukocytes and leukemic blasts, and functions to mediate trafficking of these cells within and out of the bone marrow ${ }^{[17]}$. Importantly, aberrant expression of VLA-4 in leukemic blasts has been associated with chemoresistance and poor clinical outcomes. For example, amongst patients enrolled on the Children's Oncology Group (COG) B-ALL trial P9906 who were minimal residual disease (MRD) positive following one month of induction chemotherapy, those with low $\alpha 4$ integrin expression had significantly improved overall survival (OS) relative to those with high $\alpha 4$ integrin expression ${ }^{[18]}$. Another analysis of samples from children with relapsed B-ALL enrolled on the ALL-REZ Berlin-Frankfurt-Münster (BFM) 2002 trial from the BFM study group demonstrated that patients with increased VLA-4 expression at the time of first relapse had a significantly impaired response to chemotherapy and inferior event-free survival and $\mathrm{OS}^{[19]}$. The authors of this study went on to demonstrate that VLA-4 blocking antibodies attenuated the adhesion of B-ALL cells to BMSCs in vitro, and that this was sufficient to decrease cellular proliferation and overcome the cytarabine resistance conferred by co-culture with $\mathrm{BMSCs}^{[19]}$.

In healthy hematopoietic cells, VLA-4 demonstrates high affinity for vascular cell adhesion molecule-1 (VCAM-1), a cell surface protein that is highly expressed on the vascular endothelium, as well as the ECM glycoproteins fibronectin and osteopontin ${ }^{[20]}$. In particular, it is this affinity for VCAM-1 that is thought to play the predominant role in hematopoietic cell trafficking, as antibodies against VCAM-1 are sufficient to reduce progenitor cell adhesion to BMSCs both in vitro ${ }^{[21]}$ and in vivo ${ }^{[22]}$, while anti-fibronectin antibodies are not ${ }^{[23]}$. In the context of leukemia, the VLA-4/VCAM-1 interaction is similarly thought to be the primary mediator of chemoresistance. Co-culture of ALL cell lines with BMSCs, but not with BMSC conditioned media, significantly reduced sensitivity to cytarabine and etoposide, an effect that was abrogated in the presence of VCAM-1 blocking antibodies ${ }^{[24]}$.

Mechanistically, engagement of the VLA-4/VCAM-1 axis has been shown to activate pro-survival signaling pathways in ALL cells that in turn mediate chemoresistance. For example, in vitro stimulation of VLA-4 was shown to activate the PI3K/Akt signaling pathway in a B-ALL cell line, concomitant with the induction of adriamycin resistance, and inhibition of PI3K was sufficient to restore adriamycin sensitivity ${ }^{[25]}$. Furthermore, Astier et al. ${ }^{[26]}$ analyzed global gene expression changes in ALL cells following in vitro engagement of VLA-4 and found that a number of apoptotic regulators, including members of the caspase family, are significantly downregulated following VLA-4 activation while pro-survival factors, such as XIAP and survivin, are upregulated, together contributing to a chemoresistant state. Interestingly, there is also evidence to suggest that chemoresistance is mediated by reciprocal signaling through the VLA-4/VCAM-1 axis. Jacamo et al. ${ }^{[27]}$ demonstrated that co-culture of BMSCs with leukemic blasts resulted in an upregulation of $\mathrm{NF}-\mathrm{\kappa B}$ signaling in the BMSCs themselves, and that this was attenuated in the presence of a VLA-4 blocking antibody. Furthermore, genetic or chemical inhibition of NF- $\mathrm{B}$ signaling in BMSCs restored sensitivity to vincristine in ALL cells both in vitro and in vivo. The authors speculate that the paracrine effects of many $\mathrm{NF}-\mathrm{\kappa B}$ target genes, including cytokines, that are induced in the presence of ALL cells may in turn facilitate the protective microenvironment provided by the BMSCs. Finally, VLA-4 may promote chemoresistance via its interactions with ion channels present at the leukemic cell surface. In particular, the potassium channel Kv11.1 has been shown to be overexpressed in ALL cell lines and patient samples ${ }^{[28]}$. Intriguingly, VLA-4 can 
form cell surface signaling complexes involving Kv11.1, that in turn lead to activation of ERK and PI3K/Akt signaling with the concomitant induction of chemoresistance ${ }^{[29]}$.

Given the well-established role of VLA-4 as a mediator of chemoresistance in ALL cells, there has been increasing interest in augmenting chemosensitivity through the use of anti-VLA-4 therapeutics, many of which have been used successfully in a number of autoimmune and inflammatory conditions due to their ability to modulate leukocyte activity. Natalizumab is a humanized monoclonal antibody that targets the $\alpha 4$ integrin subunit, thereby inhibiting VLA-4 and the closely related $\alpha 4 \beta 7$ integrin, which similarly binds VCAM- ${ }^{[30]}$. Hsieh et al ${ }^{[18]}$ demonstrated that natalizumab significantly prolonged survival in a xenograft model of B-ALL when combined with multi-agent chemotherapy consisting of vincristine, dexamethasone, and L-asparaginase relative to chemotherapy alone. Other therapeutic approaches involve peptide or nonpeptide ligands that compete with VCAM-1 for VLA-4 binding. One such small molecule, TBC3486, has significantly increased affinity for VLA-4 relative to $\alpha 4 \beta 7$ and has been shown to enhance chemosensitivity in vitro in B-ALL cells co-cultured with BMSCs and in vivo in a xenograft model of B-ALL ${ }^{[31]}$.

In addition to VLA-4, several other integrins have been implicated in chemoresistance in ALL. For example, $\mathrm{CD} 11 \mathrm{~b}$ is an $\alpha$ integrin that is typically expressed on myeloid cells and plays an important role in cellular migration and extravasation during an immune response ${ }^{[32]}$. While expression in lymphoid cells is usually restricted to memory B-cells, Rhein et al. ${ }^{[33]}$ investigated the significance of aberrant CD11b expression on pre-B-ALL blasts. In this analysis, the authors demonstrated that high CD11b expression at diagnosis was an independent poor prognostic factor, with the CD11b-high patients demonstrating significantly higher rates of post-induction MRD relative to the CD11b-low patients. Furthermore, they demonstrated that the blasts remaining at the end of induction therapy had significantly higher CD11b expression relative to the cells analyzed prior to the initiation of therapy. Further studies are needed to assess the functional significance of increased CD11b expression on B-ALL cells. Finally, in T-ALL cell lines and patient samples, the collagenbinding $\alpha 2 \beta 1$ integrin has been shown to confer resistance to doxorubicin. Engagement of the $\alpha 2 \beta 1$ integrin with collagen resulted in increased activity of the MAPK signaling pathway and sustained expression of the anti-apoptotic protein Mcl-1. Consistent with these findings, doxorubicin sensitivity could be restored with MEK inhibition ${ }^{[34]}$.

\section{Cadherins}

First described for their role in regulating epithelial-to-mesenchymal transitions during normal embryonic development, cadherins have since been recognized as a structurally and functionally diverse superfamily of transmembrane proteins that play important roles in normal cellular processes and in the development and progression of cancer $^{[35]}$. The intracellular domains of cadherin proteins interact with a multitude of signal transduction effectors to carry out a variety of cellular processes. One crucial class of intracellular effectors is the catenin family of proteins, which provide a physical link between the intracellular domains of cadherin proteins and the actin cytoskeleton, thereby facilitating the role of cadherins in maintaining cell adhesion. In immune cells, the $\beta$-catenin protein, through its involvement in canonical Wnt pathway signaling, plays a critical role in the regulation of immune processes ${ }^{[36]}$.

Canonical Wnt signaling involves the binding of Wnt1 ligands to their receptor, known as Frizzled (Frz). In the absence of ligand binding, the intracellular $\beta$-catenin destruction complex is stabilized, resulting in low levels of intracellular $\beta$-catenin. Upon ligand binding, this complex becomes destabilized, allowing for an increase in levels of $\beta$-catenin protein in the cytoplasm. This $\beta$-catenin protein then translocates to the nucleus and associates with the TCF/LEF transcription factor complex, converting it from a repressive complex to an activating complex, which in turn activates a transcriptional program that mediates processes such as cell proliferation and differentiation ${ }^{[37]}$. 
Of the cadherin subfamilies, the Fat cadherins are the most commonly mutated in ALL, and many reports have demonstrated their tumor suppressive activity. In an analysis of primary T-ALL samples from adult patients, Neumann et al. ${ }^{[38]}$ determined that $12 \%$ of the samples in their cohort had missense or nonsense mutations in $F A T 1$, leading to loss of expression. Similar FAT1 mutations have subsequently been reported in pediatric T-ALL ${ }^{[4]}$. Interestingly, loss of function mutations in FAT1 have been associated with augmented Wnt pathway signaling. Specifically, Morris et al.$^{[39]}$ demonstrated that the tumor suppressive effects of wildtype FAT1 protein derive from its ability to bind $\beta$-catenin at the periphery of the cell, thereby sequestering it in the cytoplasm and preventing its localization to the nucleus and activation of TCF/LEF complex. This aberrant activation of Wnt/ $\beta$-catenin signaling has been implicated as a mediator of chemoresistance in ALL. Upon exposure of ALL cell lines and primary patient samples to BMSCs, Yang et al ${ }^{[40]}$ found that the resulting cytarabine resistance was associated with inactivation of the $\beta$-catenin destruction complex and upregulation of members of the Wnt signaling pathway. Inhibition of $\beta$-catenin was sufficient to restore chemosensitivity both in vitro and in vivo. An integrated analysis of pairs of diagnostic and relapsed pediatric ALL samples incorporating gene expression, copy number alterations, and DNA methylation analyses revealed that dysregulation of this pathway was enriched at the time of disease relapse ${ }^{[41]}$, providing correlative evidence that altered Wnt/ $\beta$-catenin signaling may be a clinically relevant mediator of resistance to chemotherapy. This same group went on to functionally test this interaction by demonstrating that, in contrast to their corresponding diagnostic samples, relapsed samples had significant overactivation of Wnt/ $\beta$-catenin signaling and were highly refractory to the glucocorticoid prednisolone. In this context, the Wnt inhibitor iCRT14 synergized with prednisolone to induce apoptosis in relapsed patient samples ${ }^{[42]}$.

Intriguingly, other reports suggest that Fat cadherins may instead function as oncogenes, where they contribute to disease progression and chemoresistance. For example, de Bock et al. ${ }^{[43]}$ demonstrated that ALL cell lines express higher levels of FAT1 relative to healthy blood or bone marrow cells. The authors also demonstrated in two cohorts of pediatric patients with B-ALL that high FAT1 expression was associated with inferior relapse-free and OS. The same authors subsequently found that some T-ALL cell lines and patient samples express a truncated version of the FAT1 transcript, which in turn results in expression of a truncated protein that lacks the extracellular domain. Furthermore, this protein was found to cooperate with the Notch signaling pathway, described below, thereby serving an oncogenic function ${ }^{[4]}$.

\section{Galectins}

The lectin family of cell surface proteins recognizes and binds to carbohydrates, leading to downstream signal transduction events that play crucial roles in normal physiology and in a variety of disease states ${ }^{[4]}$. The galectins represent a subclass of lectins with binding specificity for $\beta$-galactoside epitopes, commonly found in association with proteins that undergo glycosylation during their trafficking through the secretory pathway. As a result of their distinct affinities for specific glycoproteins, galectins provide a means of interpreting the information contained within these glycosylation events and converting that information into intracellular signal transduction processes. The ability of galectins to bind their glycoprotein ligands is facilitated in part by their ability to form higher order multimeric structures, thereby increasing their binding capacity ${ }^{[46]}$. In particular, galectin-3, which is commonly overexpressed in cancer, has extensive oligomerization capabilities, allowing it to form dynamic lattice structures at the cell surface that modulate the movement and activity of its glycoprotein binding partners ${ }^{[47]}$.

Galectin-3 was first identified as a potential mediator of chemoresistance in ALL cells through an analysis of gene expression changes following co-culture of ALL cells with BMSCs. Jurkat T-ALL cells were rendered resistant to doxorubicin upon co-culture with BMSCs, concomitant with upregulation of galectin-3. Forced overexpression of galectin-3 in these cells was sufficient to confer chemoresistance in the absence of BMSCs ${ }^{[48]}$. Consistent with these findings, Fei et al. ${ }^{[49]}$ found that in vitro, patient-derived B-ALL cells harvested from underneath a BMSC feeder cell layer, where they maintained direct cell-cell contact with 
feeder cells, had more galectin-3 on their cell surface relative to cells found in suspension above the cell layer or cells cultured in the absence of BMSCs. These same authors went on to assess secreted galectin-3 levels in the growth medium from BMSCs cultured in the absence or presence of ALL cells, and found that BMSCs secreted more galectin-3 in the context of ALL cell co-culture relative to culture of BMSCs alone, while ALL cells alone did not secrete galectin-3 under any conditions. These results suggested that the elevated galectin-3 found on the cell surface of ALL cells had a stromal origin. The authors further confirmed this by demonstrating that exosomes derived from BMSCs, but not from ALL cells, contained galectin-3 and that these exosomes were secreted by BMSCs and subsequently taken up by ALL cells ${ }^{[50]}$. Hu et al ${ }^{[50]}$ similarly demonstrated that co-culture of ALL cells with BMSCs resulted in increased cell surface galectin-3 and chemoresistance, and extended this finding to demonstrate that transcriptional targets of the Wnt/ $\beta$-catenin signaling pathway, previously implicated in chemoresistance, were upregulated in wild-type ALL cells cocultured with BMSCs but not in galectin-3 knockout cells, suggesting that activation of this pathway is galectin-3-dependent ${ }^{[51]}$. Interestingly, galectin-3 inhibitors have demonstrated efficacy in acute myeloid leukemia ${ }^{[52]}$, diffuse large B-cell lymphoma ${ }^{[53]}$, and multiple myeloma ${ }^{[54,55]}$. Additional studies are necessary to determine whether such inhibitors may similarly augment chemosensitivity in the context of ALL.

\section{Notch Signaling}

The Notch signal transduction pathway is unique in several ways. First, its activation is dependent upon direct cell-cell contact between a ligand-expressing cell, such as a T-cell, and a receptor-expressing cell, such as a BMSC. Second, unlike other non-enzymatic transmembrane receptors, upon engagement of the Notch receptor by its ligands Delta-like or Jagged, the receptor itself undergoes proteolytic cleavage. Specifically, the extracellular domain of the receptor is shed first, followed by cleavage within the transmembrane domain by the $\gamma$-secretase complex. This allows the intracellular domain of the receptor (NICD) to translocate to the nucleus and function as a transcriptional regulator ${ }^{[56]}$.

In normal physiology, Notch signaling plays a critical role in T- and B-cell development. In T-cells, Notch activity is required for cell survival and proliferation during the double negative (DN) stages of thymocyte development, and is important for $\beta$ selection during the production of a fully rearranged $\alpha \beta$ T-cell receptor $(\mathrm{TCR})^{[57]}$. Further highlighting the importance of Notch signaling for thymocyte development, ectopic expression of Delta-like 1 in the OP9 stromal cell line is sufficient to support early T-cell differentiation ex $v i v o^{[58]}$. Importantly, aberrant Notch signaling is a common feature of T-ALL. In pediatric T-ALL, activating mutations in components of the Notch signaling pathway, including in $\mathrm{NOTCH} 1$ itself, are found in nearly $80 \%$ of diagnostic samples ${ }^{[4]}$. Though not found to be mutationally activated in B-ALL, Notch signaling does also play an important role in B-cell development where it promotes the differentiation of marginal zone and follicular zone B-cells ${ }^{[59]}$.

Interestingly, within the bone marrow microenvironment, Notch signaling has been shown to mediate chemoresistance in the setting of hypoxia. Specifically, the bone marrow is a highly hypoxic environment, with a reported oxygen saturation of only $87.5 \%$ in healthy subjects, compared to $99 \%$ in peripheral blood ${ }^{[60]}$. Upon exposure to a hypoxic environment, cells upregulate expression of the transcription factor hypoxiainducible factor-1 $\alpha$ (HIF-1 $\alpha$ ), which mediates a variety of tissue-specific transcriptional programs ${ }^{[61]}$. In solid tumor cells, it has been shown that Notch signaling is induced in response to hypoxia and functions to promote cellular motility and invasiveness ${ }^{[62]}$. Zou et al. ${ }^{[63]}$ similarly demonstrated that in the setting of hypoxia, T-ALL cells undergo HIF-1 $\alpha$-dependent Notch activation. In T-ALL cell lines, exposure to hypoxia was sufficient to confer chemoresistance, and this could be overcome with Notch silencing.

Due to the role for Notch signaling as a mediator of chemoresistance, there is interest in pharmacologically targeting the Notch signaling pathway. In addition to monoclonal antibodies targeting Notch receptors or ligands ${ }^{[64]}$, significant attention has been devoted to the preclinical and clinical development of $\gamma$ secretase 
inhibitors (GSIs). Interestingly, numerous preclinical studies have demonstrated the ability of GSIs to augment chemosensitivity ${ }^{[65-67]}$. Unfortunately, early single agent clinical trials involving GSIs were limited by severe gastrointestinal toxicity ${ }^{[68]}$. However, Real et al ${ }^{[69]}$ demonstrated through a series of elegant in vivo experiments that GSIs effectively overcome glucocorticoid resistance in T-ALL, and that concomitant exposure to glucocorticoids and GSIs abrogates the gastrointestinal toxicity associated with GSIs, thereby restoring the potential for the use of GSIs to modulate response to chemotherapy in T-ALL. This strategy was employed in a clinical trial involving relapsed or refractory pediatric solid tumors and T-cell leukemia (NCT01088763).

\section{SOLUBLE FACTORS}

In addition to direct cell-cell interactions, a number of secretory products made by cells within the bone marrow microenvironment engage with receptors on lymphoid cells to mediate functions such as cell proliferation and survival. Like the factors described above, many of these soluble products have similarly been shown to mediate chemoresistance.

\section{TNF $\alpha$}

$\mathrm{TNF} \alpha$ is a pro-inflammatory cytokine that is responsible for normal immune system homeostasis and plays numerous well-established roles in the development and maintenance of malignancy ${ }^{[70]}$. Produced by macrophages, natural killer cells, and T-cells, TNF $\alpha$ normally functions as a regulator of hematopoiesis when present in conjunction with other growth factors that similarly act to maintain the appropriate size and function of the hematopoietic compartment ${ }^{[7]}$. Interestingly, a polymorphism in the TNF gene itself, which results in higher plasma levels of TNF $\alpha$, has been shown to corelate with poor outcomes in a number of hematologic malignancies, including Hodgkin's ${ }^{[72]}$ and non-Hodgkin's lymphoma ${ }^{[73]}$. Consistent with these findings, Lauten et al. ${ }^{[7]}$ demonstrated in a cohort of children treated on BFM protocols that amongst patients with a prednisone poor response, those with TNF gene polymorphisms had a higher rate of relapse. Mechanistically, TNFRII signaling has been shown to activate the PI3K/Akt signal transduction pathway, leading to downstream pro-survival signaling. Activation of this pathway has been associated with the induction of doxorubicin resistance in ALL cell lines ${ }^{[75]}$, though further studies are needed to fully elucidate the relationship between altered TNF $\alpha$ expression and signaling and clinical outcomes for patients with ALL.

\section{$\gamma$ chain cytokines}

Consisting of interleukin (IL)-2, 4, 7, 9, 15, and 21, the common $\gamma$ chain family of cytokines exert their effects on immune cells via a receptor that contains one or more cytokine-specific receptor chains as well as the common cytokine receptor $\gamma$ chain. These multimeric receptor complexes lack intrinsic enzymatic activity and instead recruit downstream effectors via their cytoplasmic domains. Specifically, the cytokine-specific receptor chains most commonly recruit the kinase JAK1, while the $\gamma$ chain recruits JAK3. Activation of these JAK proteins results in phosphorylation of the cytoplasmic domains of the receptor subunits, which in turn creates docking sites for the STAT family of transcription factors, with STAT protein binding specificity determined by the identity of the specific receptor docking sites. Upon recruitment to activated cytokine receptors, these STAT proteins undergo JAK-mediated phosphorylation, leading to their translocation to the nucleus where they function as transcription factors to mediate target gene expression ${ }^{[76]}$. Due to their central roles in regulating the differentiation, survival, and proliferation of healthy lymphoid cells, it is not surprising that $\gamma$ chain cytokine receptors play an important role in mediating chemoresistance in ALL.

Produced by T-cells, IL-4 acts on a multitude of immune cell subtypes leading to a wide range of downstream effects. For example, IL-4 is a signature cytokine in the differentiation of naïve CD4+ T-cells into the Th2 subtype of T helper cells. In the B-cell lineage, IL-4 functions as a B-cell differentiation factor, promoting immunoglobulin isotype switching, and in the myeloid lineage, IL-4 is important for the 
development of M2 macrophages ${ }^{[76]}$. Importantly, IL-4 has been implicated in glucocorticoid resistance in both non-malignant ${ }^{[77,78]}$ and malignant T-cells. Serafin et al. ${ }^{[79]}$ discovered that in T-ALL patients with a poor initial response to the glucocorticoid prednisone, the IL4 gene was upregulated downstream of aberrant activation of LCK, a Src family non-receptor tyrosine kinase that is important for lymphocyte development and activation. The authors demonstrated that culturing glucocorticoid sensitive T-ALL cell lines in the presence of IL-4 was sufficient to confer glucocorticoid resistance. Interestingly, in conjunction with TNFo, IL-4 is also a potent inducer of VCAM-1 expression ${ }^{[80]}$, with TNF $\alpha$ and IL-4 exposure promoting the adhesion of ALL cells to bone marrow fibroblasts in culture ${ }^{[21,81]}$. As described above, the VLA-4/VCAM-1 axis has been implicated in chemoresistance in a variety of model systems.

IL-7 is produced by the stromal cells of the bone marrow and thymus and plays a crucial nonredundant role in lymphoid cell development and survival. Activation of the IL-7 receptor (IL-7R) primarily recruits the STAT5 transcription factors, which induce expression of antiapoptotic proteins such as BCL-2. Interestingly, many studies of early thymocyte development have demonstrated an interaction between IL-7R signaling, TCR signaling, and endogenous glucocorticoid exposure that is critical for the appropriate development of the T-cell repertoire. Specifically, in normal T-cell development, IL-7R is expressed at high levels in the early $\mathrm{DN}$ thymocyte population, thereby maintaining cell survival prior to rearrangement of the $\mathrm{TCR}^{[76]}$. Upon induction of TCR rearrangement during the late DN and early double positive (DP) stage of development, IL$7 \mathrm{R}$ is transiently downregulated, thereby abrogating its strong pro-survival signal ${ }^{[82]}$. At this stage, exposure to endogenous glucocorticoids is sufficient to induce cell death unless the cell is rescued by pro-survival signaling following successful TCR rearrangement ${ }^{[83]}$. In this model, loss of IL-7R-mediated pro-survival signaling is required to enable an apoptotic response to endogenous glucocorticoids in the absence of an appropriately rearranged TCR. In the context of T-ALL, signaling through the IL-7R/JAK/STAT5 pathway has been shown to confer resistance to pharmacologic concentrations of glucocorticoids, suggesting that this normal developmental process is co-opted in this malignant state to promote chemotherapy resistance.

Specifically, gain-of-function mutations in the IL-7R/JAK/STAT5 pathway occur in $25 \%$ of pediatric and young adult T-ALLs ${ }^{[4]}$, underscoring the strong selective pressure for activation of this pathway as a mediator of T-cell survival. Both mutational and non-mutational activation of this pathway have been implicated in glucocorticoid resistance in T-ALL. For example, Li et al. ${ }^{[10]}$ demonstrated in diagnostic samples from children with T-ALL that mutations in the IL-7R/JAK/STAT5 pathway were associated with decreased ex vivo prednisolone sensitivity and with inferior relapse free survival. The authors went on to express mutant alleles of IL-7R pathway genes in glucocorticoid sensitive T-ALL cell lines and found that many of these mutations were sufficient to confer resistance to glucocorticoids, but not to other chemotherapies. Consistent with this idea, we previously demonstrated that IL-7-induced glucocorticoid resistance most commonly occurs in T-ALLs that lack activating mutations in the IL-7R/JAK/STAT5 pathway, and that this resistance phenotype is enriched in T-ALLs of the early T-cell precursor (ETP) subset, which correspond to the early DN stages of thymocyte development. Like Li et al. ${ }^{[10]}$, we similarly found that IL-7-mediated effects on chemoresistance are specific to glucocorticoids ${ }^{[84]}$. We later showed that glucocorticoid resistance is mediated specifically by induction of the anti-apoptotic protein BCL-2 downstream of STAT5, and can be overcome with inhibition of JAK. Interestingly, we further demonstrated that this mechanism of IL-7-induced glucocorticoid resistance occurs in distinct subpopulations of healthy developing thymocytes both in vivo and ex vivo, and that T-ALLs with gene expression signatures resembling early stages of thymocyte development are enriched for this resistance phenotype ${ }^{[85]}$. Taken together, these data provide strong evidence for a developmentallyretained mechanism by which T-ALLs arising from stages of thymocyte development that rely on IL$7 \mathrm{R}$ signaling can utilize this pathway to resist apoptosis in response to glucocorticoid therapy even in the absence of activating pathway mutations. 
While not a $\gamma$ chain cytokine receptor, the thymic stromal lymphopoietin (TSLP) receptor (TSLP-R) consists of a heterodimer of the IL-7R $\alpha$ chain and cytokine receptor-like factor 2 (CRLF2), and plays a role in normal B-cell development ${ }^{[8]}$. Aberrant signaling through TSLP-R, most commonly mediated by chromosomal rearrangements of $C R L F 2$ and/or mutational activation of its downstream effector, JAK2, is a hallmark feature of Philadelphia chromosome-like (Ph-like) B-ALL. These leukemias demonstrate a gene expression signature that is similar to that of Ph-positive B-ALL but lack the BCR-ABL1 translocation ${ }^{[87]}$. Importantly, CRLF2 rearrangements and JAK2 mutations, collectively leading to increased TSLP-R signaling capacity, have been associated with poor outcomes in patients with Ph-like $\mathrm{ALL}^{[88]}$. Similar to T-ALL, we previously demonstrated that CRLF2-rearranged Ph-like B-ALL samples uniformly demonstrate in vitro glucocorticoid resistance ${ }^{[89]}$.

Given the role of cytokine-mediated JAK/STAT signaling in the pathogenesis and chemoresistance of ALL cells, there has been considerable interest in targeting this pathway as a means of improving clinical outcomes. While several approaches have been studied, including the use of reducing agents to disrupt disulfide bond formation within IL-7R, blocking antibodies against IL-7R, and IL-7R-directed chimeric antigen receptor (CAR) T-cells ${ }^{[90]}$, small molecule inhibitors of JAK are currently furthest along in clinical development. Ruxolitinib is a JAK1/2 inhibitor that has shown efficacy as a single agent in xenograft models of ETP T-ALL ${ }^{[91]}$ and Ph-like ALL ${ }^{[92]}$, and has been shown to sensitize ALL cells to glucocorticoids in vitro ${ }^{[84,85,89]}$. Consistent with these promising results, clinical trials have been initiated to investigate the efficacy of ruxolitinib in ALL (NCT03571321 and NCT02723994).

\section{Chemokines}

Chemokines are a subclass of cytokines that are specifically responsible for the export of immune cells from the bone marrow and for the homing of these cells to sites of infection and inflammation. Chemokine ligands characteristically contain one or more cysteine residues, enabling their division into the $\mathrm{XC}$, CC, $\mathrm{CXC}, \mathrm{CX} 3 \mathrm{C}$ subgroups on the basis of the location of their cysteine residues within their amino acid sequences. Upon secretion, chemokines bind to G-protein-coupled receptors, with some receptors showing specificity for a single ligand and others capable of binding multiple distinct ligands ${ }^{[93]}$. In particular, CXCR4 signaling has been implicated in the initiation and progression of ALL, and has further been shown to mediate chemoresistance.

CXCL12, also called stromal derived factor 1 (SDF-1) is the chemokine ligand for the CXCR4 receptor and is expressed ubiquitously at all stages of development, where it plays an important role in tissue homeostatic functions, including within the hematopoietic compartment ${ }^{[94]}$. Importantly, aberrant CXCL12/CXCR4 signaling has been functionally implicated in leukemogenesis and associated with poor clinical outcomes. For example, it has been shown that the protein phosphatase calcineurin is aberrantly activated in T-ALL and is required for the activity of leukemia initiating cells ${ }^{[95]}$. Passaro et al. ${ }^{[96]}$ further demonstrated that cell surface expression of CXCR4 is regulated by calcineurin activity, and the presence of CXCR4 at the cell surface is required for the migratory capability of T-ALL cells. The authors further showed that silencing of CXCR4 was associated with the induction of apoptosis in a T-ALL cell line and in a patient-derived xenograft model of T-ALL. In pediatric B-ALL, high CXCR4 expression was associated with inferior relapse free survival $^{[97]}$, and in adult B-ALL, phosphorylated CXCR4, corresponding to an active form of the receptor, was associated with reduced $\mathrm{OS}^{[98]}$.

To assess the relationship between CXCR4 expression and chemosensitivity in ALL, Sison et al. ${ }^{[99]}$ utilized primary samples from infants with mixed lineage leukemia $(M L L)$-rearranged ALL, a high-risk molecular subtype of B-ALL that is associated with poor outcomes. Consistent with other reports, the authors found that exposure to BMSCs conferred chemoresistance and that co-treatment with the CXCR4 antagonist plerixafor was sufficient to restore chemosensitivity. The same group went on to demonstrate that in response 
to chemotherapy, ALL cells upregulate expression of CXCR4, priming them to respond to BMSC-secreted CXCL12. In light of these findings, the authors demonstrated the efficacy of a combinatorial therapeutic strategy involving plerixafor in an in vivo xenograft model and found that co-treatment with plerixafor abrogated the protective effect of the bone marrow microenvironment on the response to chemotherapy ${ }^{[100]}$. As a result of these promising results in preclinical models, the Pediatric Oncology Experimental Therapeutics Investigators' consortium conducted a phase 1 study to evaluate the potential for combining plerixafor with the chemotherapies cytarabine and etoposide in children with relapsed or refractory acute leukemia (NCT01319864). While the only clinical responses observed in this cohort occurred in children with AML, the number of patients with ALL was small ${ }^{[101]}$. Based on these results, the role of plerixafor in augmenting chemosensitivity remains to be studied in additional patient cohorts in conjunction with other chemotherapy backbones.

\section{Asparagine}

The introduction of L-asparaginase into modern treatment regimens has dramatically improved outcomes for patients with $A L L{ }^{[102]}$. The efficacy of $\mathrm{L}$-asparaginase relies upon the unique requirement for asparagine in leukemia cells. In healthy cells, asparagine is a non-essential amino acid, synthesized intracellularly through the activity of asparagine synthetase. In contrast, ALL cells lack sufficient expression of asparagine synthetase to meet their requirement for asparagine, thereby relying instead on exogenous sources. The use of L-asparaginase exploits this dependency by hydrolyzing asparagine, thus depriving ALL cells of this exogenous supply ${ }^{[103]}$. BMSCs have been shown to contribute to L-asparaginase resistance through upregulation of asparagine synthetase. Specifically, Iwamoto et al. ${ }^{[104]}$ demonstrated that expression of the gene encoding asparagine synthetase, ASNS, was significantly higher in BMSCs than in ALL cells themselves, and that co-culture of ALL cells with BMSCs conferred resistance to L-asparaginase, suggesting that BMSCs function as an exogenous source of asparagine for ALL cells.

\section{CONCLUSION}

Comprehensive analyses of the genomic and transcriptomic landscape of ALL have revealed many factors that contribute to chemoresistance and poor clinical outcomes for patients with ALL. In contrast to these cell-intrinsic drivers of chemoresistance, cell-extrinsic factors are less well-appreciated for their role in modulating clinical outcomes. As described here, the bone marrow microenvironment, through direct cellcell interactions and the production of soluble factors, plays a complex and multifactorial role in determining the response of ALL cells to chemotherapy. This review highlights a number of efforts to target the microenvironment as a means of augmenting the efficacy of chemotherapy. While many of the preclinical studies described here present promising results, further studies are needed to validate these therapeutic strategies in other preclinical model systems and to facilitate their eventual clinical implementation.

\section{DECLARATIONS}

\section{Author contributions}

Wrote and edited the manuscript: Meyer LK

Wrote and edited the manuscript: Hermiston ML

\section{Availability of data and materials}

Not applicable.

\section{Financial support and sponsorship}

Meyer LK is supported by a Genentech Foundation Fellowship Award. Hermiston ML is supported by the National Cancer Institute Grant R01 CA193776, the Buster Posey Family Foundation, the Campini Foundation, and the Pepp Family Foundation. 


\section{Conflicts of Interest}

All authors declare that there are no conflicts of interest.

\section{Ethical approval and consent to participate}

Not applicable.

\section{Consent for publication}

Not applicable.

\section{Copyright}

(c) The Author(s) 2019.

\section{REFERENCES}

1. Pui CH, Mullighan CG, Evans WE, Relling MV. Pediatric acute lymphoblastic leukemia: where are we going and how do we get there? Blood 2012;120:1165-74.

2. Ronson A, Tvito A, Rowe JM. Treatment of relapsed/refractory acute lymphoblastic leukemia in adults. Curr Oncol Rep 2016;18:39.

3. Liu YF, Wang BY, Zhang WN, Huang JY, Li BS, et al. Genomic profiling of adult and pediatric B-cell acute lymphoblastic leukemia. EBioMedicine 2016;8:173-83.

4. Liu Y, Easton J, Shao Y, Maciaszek J, Wang Z, et al. The genomic landscape of pediatric and young adult T-lineage acute lymphoblastic leukemia. Nat Genet 2017;49:1211-8.

5. De Keersmaecker K, Atak ZK, Li N, Vicente C, Patchett S, et al. Exome sequencing identifies mutation in CNOT3 and ribosomal genes RPL5 and RPL10 in T-cell acute lymphoblastic leukemia. Nat Genet 2013;45:186-90.

6. Lugthart S, Cheok MH, den Boer ML, Yang W, Holleman A, et al. Identification of genes associated with chemotherapy crossresistance and treatment response in childhood acute lymphoblastic leukemia. Cancer Cell 2005;7:375-86.

7. Holleman A, Cheok MH, den Boer ML, Yang W, Veerman AJP, et al. Gene-expression patterns in drug-resistant acute lymphoblastic leukemia cells and response to treatment. N Engl J Med 2004;351:533-42.

8. Stam RW, Den Boer ML, Schneider P, de Boer J, Hagelstein J, et al. Association of high-level MCL-1 expression with in vitro and in vivo prednisone resistance in MLL-rearranged infant acute lymphoblastic leukemia. Blood 2010;115:1018-25.

9. Piovan E, Yu J, Tosello V, Herranz D, Ambesi-Impiombato A, et al. Direct reversal of glucocorticoid resistance by AKT inhibition in acute lymphoblastic leukemia. Cancer Cell 2013;24:766-76.

10. Li Y, Buijs-Gladdines JGCAM, Canté-Barrett K, Stubbs AP, Vroegindeweij EM, et al. IL-7 receptor mutations and steroid resistance in pediatric T cell acute lymphoblastic leukemia: a genome sequencing study. PLoS Med 2016;13:e1002200.

11. Bakker E, Qattan M, Mutti L, Demonacos C, Krstic-Demonacos M. The role of microenvironment and immunity in drug response in leukemia. Biochim Biophys Acta 2016;1863:414-26.

12. Konopleva M, Tabe $\mathrm{Y}$, Zeng Z, Andreeff M. Therapeutic targeting of microenvironmental interactions in leukemia: mechanisms and approaches. Drug Resist Updat 2009;12:103-13.

13. Tavor S, Petit I. Can inhibition of the SDF-1/CXCR4 axis eradicate acute leukemia? Semin Cancer Biol 2010;20:178-85.

14. Konopleva MY, Jordan CT. Leukemia stem cells and microenvironment: biology and therapeutic targeting. Journal of clinical oncology 2011;29:591-9.

15. Manabe A, Coustan-Smith E, Behm FG, Raimondi SC, Campana D. Bone marrow-derived stromal cells prevent apoptotic cell death in B-lineage acute lymphoblastic leukemia. Blood 1992;79:2370-7.

16. Manabe A, Murti KG, Coustan-Smith E, Kumagai M, Behm FG, et al. Adhesion-dependent survival of normal and leukemic human B lymphoblasts on bone marrow stromal cells. Blood 1994;83:758-66.

17. Takada Y, Ye X, Simon S. The integrins. Genome Biol 2007;8:215.

18. Hsieh YT, Gang EJ, Geng H, Park E, Huantes S,et al. Integrin alpha4 blockade sensitizes drug resistant pre-B acute lymphoblastic leukemia to chemotherapy. Blood 2013;121:1814-8.

19. Shalapour S, Hof J, Kirschner-Schwabe R, Bastian L, Eckert C, et al. High VLA-4 expression is associated with adverse outcome and distinct gene expression changes in childhood B-cell precursor acute lymphoblastic leukemia at first relapse. Haematologica 2011;96:1627-35.

20. Shishido S, Bönig H, Kim YM. Role of integrin alpha4 in drug resistance of leukemia. Front Oncol 2014;4:99.

21. Bradstock K, Makrynikola V, Bianchi A, Byth K. Analysis of the mechanism of adhesion of precursor-B acute lymphoblastic leukemia cells to bone marrow fibroblasts. Blood 1993;82:3437-44.

22. Papayannopoulou T, Craddock C, Nakamoto B, Priestley GV, Wolf NS. The VLA4/VCAM-1 adhesion pathway defines contrasting mechanisms of lodgement of transplanted murine hemopoietic progenitors between bone marrow and spleen. Proc Natl Acad Sci USA 1995;92:9647-51.

23. Craddock CF, Nakamoto B, Elices M, Papayannopoulou Th. The role of CS1 moiety of fibronectin in VLA mediated haemopoietic progenitor trafficking. Br J Haematol 1997;97:15-21.

24. Mudry RE, Fortney JE, York T, Hall BM, Gibson LF. Stromal cells regulate survival of B-lineage leukemic cells during chemotherapy. 
Blood 2000;96:1926-32.

25. Sarkar S, Svoboda M, de Beaumont R, Freedman AS. The role of Aktand RAFTK in beta1 integrin mediated survival of precursor B-acute lymphoblastic leukemia cells. Leuk Lymphoma 2002;43:1663-71.

26. Astier AL, Xu R, Svoboda M, Hinds E, Munoz O, et al, Temporal gene expression profile of human precursor B leukemia cells induced by adhesion receptor: identification of pathways regulating B-cell survival. Blood 2003;101:1118-27.

27. Jacamo R, Chen Y, Wang Z, Ma W, Zhang M,et al. Reciprocal leukemia-stroma VCAM-1/VLA-4-dependent activation of NF- $\mathrm{B}$ mediates chemoresistance. Blood 2014;123:2691-702.

28. Arcangeli A, Pillozzi S, Becchetti A. Targeting ion channels in leukemias: a new challenge for treatment. Curr Med Chem 2012;19:683-96.

29. Pillozzi S, Masselli M, De Lorenzo E, Accordi B, Cilia E, et al. Chemotherapy resistance in acute lymphoblastic leukemia requires hERG1 channels and is overcome by hERG1 blockers. Blood 2011;117:902-14.

30. Kapp TG, Rechenmacher F, Sobahi TR, Kessler H. Integrin modulators: a patent review. Expert Opin Ther Pat 2013;23:1273-95.

31. Hsieh YT, Gang EJ, Shishido SN, Kim HN, Pham J, et al. Effects of the small-molecule inhibitor of integrin $\alpha 4$, TBC3486, on pre-BALL cells. Leukemia 2014;28:2101-4.

32. Fagerholm SC, Varis M, Stefanidakis M, Hilden TJ, Gahmberg CG. Alpha-chain phosphorylation of the human leukocyte CD11b/ CD18 (Mac-1) integrin is pivotal for integrin activation to bind ICAMs and leukocyte extravasation. Blood 2006;108:3379-86.

33. Rhein P, Mitlohner R, Basso G, Gaipa G, Dworzak MN, et al. CD11b is a therapy resistance- and minimal residual disease-specific marker in precursor B-cell acute lymphoblastic leukemia. Blood 2010;115:3763-71.

34. Naci D, El Azreq MA, Chetoui N, Lauden L, Sigaux F, et al. $\alpha 2 \beta 1$ integrin promotes chemoresistance against doxorubicin in cancer cells through extracellular signal-regulated kinase (ERK). J Biol Chem 2012;287:17065-76.

35. Angst BD, Marcozzi C, Magee AI. The cadherin superfamily: diversity in form and function. J Cell Sci 2001;114:629-41.

36. Heuberger J, Birchmeier W. Interplay of cadherin-mediated cell adhesion and canonical Wnt signaling. Cold Spring Harb Perspect Biol 2010;2:a002915.

37. Chae WJ, Bothwell ALM. Canonical and non-canonical wnt signaling in immune cells. Trends Immunol 2018;39:830-47.

38. Neumann M, Seehawer M, Schlee C, Vosberg S, Heesch S,et al. FAT1 expression and mutations in adult acute lymphoblastic leukemia. Blood Cancer J 2014;4:e224.

39. Morris LGT, Kaufman AM, Gong Y, Ramaswami D, Walsh LA, et al. Recurrent somatic mutation of FAT1 in multiple human cancers leads to aberrant Wnt activation. Nat Genet 2013;45:253-61.

40. Yang Y, Mallampati S, Sun B, Zhang J, Kim S-B, et al. Wnt pathway contributes to the protection by bone marrow stromal cells of acute lymphoblastic leukemia cells and is a potential therapeutic target. Cancer Lett 2013;333:9-17.

41. Hogan LE, Meyer JA, Yang J, Wang J, Wong N, et al. Integrated genomic analysis of relapsed childhood acute lymphoblastic leukemia reveals therapeutic strategies. Blood 2011;118:5218-26.

42. Dandekar S, Romanos-Sirakis E, Pais F, Bhatla T, Jones C, et al. Wnt inhibition leads to improved chemosensitivity in paediatric acute lymphoblastic leukaemia. Br J Haematol 2014;167:87-99.

43. de Bock CE, Ardjmand A, Molloy TJ, Bone SM, Johnstone D, et al. The Fat1 cadherin is overexpressed and an independent prognostic factor for survival in paired diagnosis-relapse samples of precursor B-cell acute lymphoblastic leukemia. Leukemia 2012;26:918-26.

44. de Bock CE, Down M, Baidya K, Sweron B, Boyd AW, et al. T-cell acute lymphoblastic leukemias express a unique truncated FAT1 isoform that cooperates with NOTCH1 in leukemia development. Haematologica 2019;104:e204-e207.

45. Yau T, Dan X, Ng CCW, Ng TB. Lectins with potential for anti-cancer therapy. Mol Basel Switz 2015;20:3791-810.

46. Thijssen VL, Heusschen R, Caers J, Griffioen AW. Galectin expression in cancer diagnosis and prognosis: a systematic review. Biochim Biophys Acta 2015;1855:235-47.

47. Nabi IR, Shankar J, Dennis JW. The galectin lattice at a glance. J Cell Sci 2015;128:2213-9.

48. Yamamoto-Sugitani M, Kuroda J, Ashihara E, Nagoshi H, Kobayashi T,et al. Galectin-3 (Gal-3) induced by leukemia microenvironment promotes drug resistance and bone marrow lodgment in chronic myelogenous leukemia. Proc Natl Acad Sci USA 2011;108:17468-73.

49. Fei F, Abdel-Azim H, Lim M, Arutyunyan A, von Itzstein M, et al. Galectin-3 in pre-B acute lymphoblastic leukemia. Leukemia 2013;27:2385-8

50. Fei F, Joo EJ, Tarighat SS, Schiffer I, Paz H, et al. B-cell precursor acute lymphoblastic leukemia and stromal cells communicate through Galectin-3. Oncotarget 2015;6:11378-94.

51. Hu K, Gu Y, Lou L, Liu L, Hu Y, et al. Galectin-3 mediates bone marrow microenvironment-induced drug resistance in acute leukemia cells via Wnt/ $\beta$-catenin signaling pathway. J Hematol OncolJ Hematol Oncol 2015;8:1.

52. Ruvolo PP, Ruvolo VR, Benton CB, AlRawi A, Burks JK, et al. Combination of galectin inhibitor GCS-100 and BH3 mimetics eliminates both p53 wild type and p53 null AML cells. Biochim Biophys Acta 2016;1863:562-71.

53. Clark MC, Pang M, Hsu DK, Liu FT, de Vos S, et al. Galectin-3 binds to CD45 on diffuse large B-cell lymphoma cells to regulate susceptibility to cell death. Blood 2012;120:4635-44.

54. Streetly MJ, Maharaj L, Joel S, Schey SA, Gribben JG, et al. GCS-100, a novel galectin-3 antagonist, modulates MCL-1, NOXA, and cell cycle to induce myeloma cell death. Blood 2010;115:3939-48.

55. Chauhan D, Li G, Podar K, Hideshima T, Neri P, et al. A novel carbohydrate-based therapeutic GCS-100 overcomes bortezomib resistance and enhances dexamethasone-induced apoptosis in multiple myeloma cells. Cancer Res 2005;65:8350-8.

56. Nwabo Kamdje AH, Krampera M. Notch signaling in acute lymphoblastic leukemia: any role for stromal microenvironment? Blood 2011;118:6506-14.

57. Li X, von Boehmer H. Notch signaling in T-Cell development and T-ALL. ISRN Hematol 2011;2011:921706.

58. Schmitt TM, Zúñiga-Pflücker JC. Induction of T cell development from hematopoietic progenitor cells by delta-like-1 in vitro. 
Immunity 2002;17:749-56.

59. Siebel C, Lendahl U. Notch signaling in development, tissue homeostasis, and disease. Physiol Rev 2017;97:1235-94.

60. Harrison JS, Rameshwar P, Chang V, Bandari P. Oxygen saturation in the bone marrow of healthy volunteers. Blood 2002;99:394.

61. Ke Q, Costa M. Hypoxia-inducible factor-1 (HIF-1). Mol Pharmacol 2006;70:1469-80.

62. Sahlgren C, Gustafsson MV, Jin S, Poellinger L, Lendahl U. Notch signaling mediates hypoxia-induced tumor cell migration and invasion. Proc Natl Acad Sci USA 2008;105:6392-7.

63. Zou J, Li P, Lu F, Liu N, Dai J, et al. Notch1 is required for hypoxia-induced proliferation, invasion and chemoresistance of T-cell acute lymphoblastic leukemia cells. J Hematol OncolJ Hematol Oncol 2013;6:3.

64. Takebe N, Nguyen D, Yang SX. Targeting notch signaling pathway in cancer: clinical development advances and challenges. Pharmacol Ther 2014;141:140-9.

65. Takam Kamga P, Dal Collo G, Midolo M, Adamo A, Delfino P, et al. Inhibition of notch signaling enhances chemosensitivity in B-cell precursor acute lymphoblastic leukemia. Cancer Res 2019;79:639-49.

66. Nwabo Kamdje AH, Mosna F, Bifari F, Lisi V, Bassi G, et al. Notch-3 and Notch-4 signaling rescue from apoptosis human B-ALL cells in contact with human bone marrow-derived mesenchymal stromal cells. Blood 2011;118:380-9.

67. Meng X, Matlawska-Wasowska K, Girodon F, Mazel T, Willman CL, et al. GSI-I (Z-LLNle-CHO) inhibits $\gamma$-secretase and the proteosome to trigger cell death in precursor-B acute lymphoblastic leukemia. Leukemia 2011;25:1135-46.

68. DeAngelo DJ, Stone RM, Silverman LB, Stock W, attar EC, et al. A phase I clinical trial of the notch inhibitor MK-0752 in patients with T-cell acute lymphoblastic leukemia/lymphoma (T-ALL) and other leukemias. Journal of Clinical Oncology 2006;24: 6585-6585.

69. Real PJ, Tosello V, Palomero T, Castillo M, Hernando E, et al. Gamma-secretase inhibitors reverse glucocorticoid resistance in T cell acute lymphoblastic leukemia. Nat Med 2009;15:50-8.

70. Balkwill F. TNF-alpha in promotion and progression of cancer. Cancer Metastasis Rev 2006;25:409-16.

71. Riether C, Schürch CM, Ochsenbein AF. Regulation of hematopoietic and leukemic stem cells by the immune system. Cell Death Differ 2015;22:187-98.

72. Warzocha K, Bienvenu J, Ribeiro P, Moullet I, Dumontet C, et al. Plasma levels of tumour necrosis factor and its soluble receptors correlate with clinical features and outcome of Hodgkin's disease patients. Br J Cancer 1998;77:2357-62.

73. Warzocha K, Ribeiro P, Bienvenu J, Roy P, Charlot C, et al. Genetic polymorphisms in the tumor necrosis factor locus influence nonHodgkin's lymphoma outcome. Blood 1998;91:3574-81.

74. Lauten M, Matthias T, Stanulla M, Beger C, Welte K, et al. Association of initial response to prednisone treatment in childhood acute lymphoblastic leukaemia and polymorphisms within the tumour necrosis factor and the interleukin-10 genes. Leukemia 2002;16:143742.

75. Gu L, Findley HW, Zhu N, Zhou M. Endogenous TNFalpha mediates cell survival and chemotherapy resistance by activating the PI3K/Akt pathway in acute lymphoblastic leukemia cells. Leukemia 2006;20:900-4.

76. Lin JX, Leonard WJ. The common cytokine receptor $\gamma$ chain family of cytokines. Cold Spring Harb Perspect Biol 2018;10:a028449.

77. Zubiaga AM, Munoz E, Huber BT. IL-4 and IL-2 selectively rescue Th cell subsets from glucocorticoid-induced apoptosis. J Immunol Baltim Md 1992;149:10712.

78. Kam JC, Szefler SJ, Surs W, Sher ER, Leung DY. Combination IL-2 and IL-4 reduces glucocorticoid receptor-binding affinity and T cell response to glucocorticoids. J Immunol Baltim Md 1993;151:3460-6.

79. Serafin V, Capuzzo G, Milani G, Minuzzo SA, Pinazza M, et al. Glucocorticoid resistance is reverted by LCK inhibition in pediatric T-cell acute lymphoblastic leukemia. Blood 2017;130:2750-61.

80. McCarty JM, Yee EK, Deisher TA, Harlan JM, Kaushansky K. Interleukin-4 induces endothelial vascular cell adhesion molecule-1 (VCAM-1) by an NF-kappa b-independent mechanism. FEBS Lett 1995;372:194-8.

81. Juneja HS, Schmalsteig FC, Lee S, Chen J. Vascular cell adhesion molecule-1 and VLA-4 are obligatory adhesion proteins in the heterotypic adherence between human leukemia/lymphoma cells and marrow stromal cells. Exp Hematol 1993;21:444-50.

82. Mazzucchelli R, Durum SK. Interleukin-7 receptor expression: intelligent design. Nat Rev Immunol 2007;7:144-54.

83. Ashwell JD, King LB, Vacchio MS. Cross-talk between the T cell antigen receptor and the glucocorticoid receptor regulates thymocyte development. Stem Cells Dayt Ohio 1996;14:490-500.

84. Delgado-Martin C, Meyer LK, Huang BJ, Shimano KA, Zinter MS, et al. JAK/STAT pathway inhibition overcomes IL7-induced glucocorticoid resistance in a subset of human T-cell acute lymphoblastic leukemias. Leukemia 2017;31:2568.

85. Meyer LK, Huang B, Roy R, Hechmer A, Anica M. et al. Glucocorticoids paradoxically induce intrinsic steroid resistance through a STAT5-mediated survival mechanism in T-cell acute lymphoblastic Leukemia. Blood 2018;132:913.

86. Ray RJ, Furlonger C, Williams DE, Paige CJ. Characterization of thymic stromal-derived lymphopoietin (TSLP) in murine B cell development in vitro. Eur J Immunol 1996;26:10-6.

87. Roberts KG, Morin RD, Zhang J, Hirst M, Zhao Y, et al. Genetic alterations activating kinase and cytokine receptor signaling in highrisk acute lymphoblastic leukemia. Cancer Cell 2012;22:153-66.

88. Harvey RC, Mullighan CG, Chen IM, Wharton W, Mikhail FM, et al. Rearrangement of CRLF2 is associated with mutation of JAK kinases, alteration of IKZF1, hispanic/latino ethnicity, and a poor outcome in pediatric B-progenitor acute lymphoblastic leukemia. Blood 2010;115:5312-21.

89. Meyer LK, Delgado-Martin C, Maude SL, Teachey DT, Hermiston ML. CRLF2 rearrangement status in ph-like all predicts intrinsic glucocorticoid resistance in vitro that is reversible with targeted MAPK and PI3K pathway inhibition. Blood 2016.

90. Cramer SD, Aplan PD, Durum SK. Therapeutic targeting of IL-7R $\alpha$ signaling pathways in ALL treatment. Blood 2016;128:473-8.

91. Maude SL, Dolai S, Delgado-Martin C, Vincent T, Robbins A, et al. Efficacy of JAK/STAT pathway inhibition in murine xenograft models of early T-cell precursor (ETP) acute lymphoblastic leukemia. Blood 2015;125:1759-67.

92. Maude SL, Tasian SK, Vincent T, Hall JW, Sheen C, et al. Targeting JAK1/2 and mTOR in murine xenograft models of ph-like acute 
lymphoblastic leukemia. Blood 2012;120:3510-8.

93. Sokol CL, Luster AD. The chemokine system in innate immunity. Cold Spring Harb Perspect Biol 2015.

94. Pozzobon T, Goldoni G, Viola A, Molon B. CXCR4 signaling in health and disease. Immunol Lett 2016;177:6-15.

95. Gachet S, Genescà E, Passaro D, Irigoyen M, Alcalde H, et al. Leukemia-initiating cell activity requires calcineurin in T-cell acute lymphoblastic leukemia. Leukemia 2013;27:2289-300.

96. Passaro D, Irigoyen M, Catherinet C, Gachet S, Da Costa De Jesus C, et al. CXCR4 is required for leukemia-initiating cell activity in T cell acute lymphoblastic leukemia. Cancer Cell 2015;27:769-79.

97. van den Berk LCJ, van der Veer A, Willemse ME, Theeuwes MJGA, Luijendijk MW, et al. Disturbed CXCR4/CXCL12 axis in paediatric precursor B-cell acute lymphoblastic leukaemia. Br J Haematol 2014;166:240-9.

98. Konoplev S, Jorgensen JL, Thomas DA, Lin E, Burger J, et al. Phosphorylated CXCR4 is associated with poor survival in adults with B-acute lymphoblastic leukemia. Cancer 2011;117:4689-95.

99. Sison EAR, Rau RE, McIntyre E, Li L, Small D,et al. MLL-rearranged acute lymphoblastic leukaemia stem cell interactions with bone marrow stroma promote survival and therapeutic resistance that can be overcome with CXCR4 antagonism. Br J Haematol 2013;160:78597.

100. Sison EAR, Magoon D, Li L, Annesley CE, Rau RE, et al. Plerixafor as a chemosensitizing agent in pediatric acute lymphoblastic leukemia: efficacy and potential mechanisms of resistance to CXCR4 inhibition. Oncotarget 2014;5:8947-58.

101. Cooper TM, Sison EAR, Baker SD, Li L, Ahmed A, et al. A phase 1 study of the CXCR4 antagonist plerixafor in combination with high-dose cytarabine and etoposide in children with relapsed or refractory acute leukemias or myelodysplastic syndrome: a pediatric oncology experimental therapeutics investigators' consortium study (POE 10-03). Pediatr Blood Cancer 2017;64:e26414.

102. Pieters R, Hunger SP, Boos J, Rizzari C, Silverman L, et al. L-asparaginase treatment in acute lymphoblastic leukemia: a focus on Erwinia asparaginase. Cancer 2011;117:238-49.

103. Kumar K, Kaur J, Walia S, Pathak T, Aggarwal D, et al. L-asparaginase: an effective agent in the treatment of acute lymphoblastic leukemia. Leuk Lymphoma 2014;55:256-62.

104. Iwamoto S, Mihara K, Downing JR, Pui CH, Campana D. Mesenchymal cells regulate the response of acute lymphoblastic leukemia cells to asparaginase. J Clin Invest 2007;117:1049-57. 